\title{
Structure of the $3^{\prime}$-hairpin of the TYMV pseudoknot: preformation in RNA folding
}

\section{Michaël H.Kolk, Marinette van der Graaf', Carel T.M.Fransen, Sybren S.Wijmenga ${ }^{2}$, Cornelis W.A.Pleij ${ }^{3}$, Hans A.Heus and Cornelis W.Hilbers ${ }^{4}$}

NSR Center for Molecular Structure, Design and Synthesis, Laboratory of Biophysical Chemistry, University of Nijmegen, Toernooiveld, 6525 ED Nijmegen and ${ }^{3}$ Leiden Institute of Chemistry, University of Leiden, PO Box 9502, 2300 RA, Leiden,

The Netherlands

${ }^{1}$ Present address: Department of Radiology, University Hospital, Nijmegen, PO Box 9101, 6500 HB Nijmegen, The Netherlands

${ }^{2}$ Present address: Department of Medical Biochemistry and

Biophysics, University of Umeå, S901 87, Sweden

${ }^{4}$ Corresponding author

e-mail: ceesh@sci.kun.nl

The solution structure of an RNA-hairpin present in the pseudoknot, which is found at the $3^{\prime}$-terminus of turnip yellow mosaic virus genomic RNA, has been solved by nuclear magnetic resonance spectroscopy. The loop, which contains the sequence 5'-GGGUCA$3^{\prime}$, was found to be highly structured and, contrary to expectations, does not attain its stability through GA or GC base pair formation but by triple interactions between the tilted adenosine and the minor groove sides of the first two guanosines. Interestingly, a very similar conformation was found for the cognate pseudoknot, implying that the $3^{\prime}$-hairpin is preformed for folding into a pseudoknotted structure. These findings suggest a mechanism of 'predetermined-fit' as a principle in RNA folding.

Keywords: hairpin/NMR/pseudoknot/RNA/TYMV

\section{Introduction}

Despite considerable progress in recent years, the number of high-resolution structures reported for RNA has remained very modest when compared with the preponderance of structures that have been determined for proteins. Nevertheless, it has already become clear that RNA structure can match that of any protein in complexity and versatility. Obviously, the building blocks of these two classes of biomolecules are markedly different. While proteins consist of two essential structural elements, $\alpha$-helices and $\beta$-strands, RNA structure has only one principal component, the double helix. Typically, the strands within double-helical stem regions are connected by loops, which can have various lengths and topologies, and some of which are highly structured.

There is mounting evidence suggesting that these loop domains in RNA can have functional as well as structural roles. A classical example of a functional role for RNA is the codon-anticodon interaction; other examples that have been discovered more recently include the interactions between proteins or peptides and RNA loops (Puglisi et al., 1992; Oubridge et al., 1994; Valegård et al., 1994; Allain et al., 1996; Battiste et al., 1996; Cai et al., 1998; Legault et al., 1998). An example of a structural role played by RNA loops is the binding between GNRA tetraloops and their cognate tetraloop receptors, as was found, for instance, in the group I intron (Cate et al., 1996).

Structurally essential loop regions are also found in RNA pseudoknots, a widely occurring motif in which the interaction between a loop and a distal single-stranded RNA region is the essential structural element. Recently, we have solved the nuclear magnetic resonance (NMR) structure of a pseudoknot that is present in the tRNA-like structure at the $3^{\prime}$-terminus of turnip yellow mosaic virus (TYMV) genomic RNA (Kolk et al., 1998). The structural role of the loops that are involved in pseudoknot formation is clearly different from the function of most other structurally relevant RNA loops, in that parts of these loops engage in Watson-Crick base pairing upon binding to the single-stranded nucleotides, while the remaining loop residues form a pseudoknot loop which may interact with its adjoining stem domain. In the TYMV pseudoknot, the loop spanning the minor groove interacts closely with the opposing helix, in particular through hydrogen bonds to a central adenosine. The delicate network of interactions around this key domain of the pseudoknot raises the question of how its constituents could bind and merge into one structural entity, particularly when compared with other examples of RNA-loop binding, such as the aforementioned binding of GNRA tetraloops.

Figure 1 shows that the triple-stranded region at the central domain of the pseudoknot is composed of a sixmembered loop region (residues $\mathrm{G} 30 \rightarrow \mathrm{A} 35$ ), called the $3^{\prime}$-hairpin loop, and a stretch of three cytidines (residues $\mathrm{C} 18 \rightarrow \mathrm{C} 20$ ), which base pair to the first three guanosines of the loop to form stem 1 of the pseudoknot. Based on its sequence, the isolated $3^{\prime}$-hairpin loop could form a sheared GA base pair, as is observed in GNRA tetraloops (Heus and Pardi, 1991) and in pentaloops with a GNRA fold (Jucker and Pardi, 1995) in boxB RNA (Cai et al., 1998; Legault et al., 1998). In fact, it has been suggested that any loop having a $\operatorname{GNR}(\mathrm{N})_{x} \mathrm{~A}(x=1,2,3, .$.$) consensus$ sequence (which includes that of the $3^{\prime}$-hairpin loop studied in the present paper) may form such a GNRA fold (Abramovitz and Pyle, 1997; Legault et al., 1998). Alternatively, C13 could engage in a Watson-Crick base pair to one of the three guanosines in the loop.

Any of these base pair interactions in the $3^{\prime}$-hairpin loop is expected to significantly stabilize its structure. Because they are incompatible with the conformation of this site in the pseudoknot, they would need to be broken in order to enable the guanosines to interact with the triple $\mathrm{C}$ sequence. The gain in free energy associated with 


\section{TYMV pseudoknot}
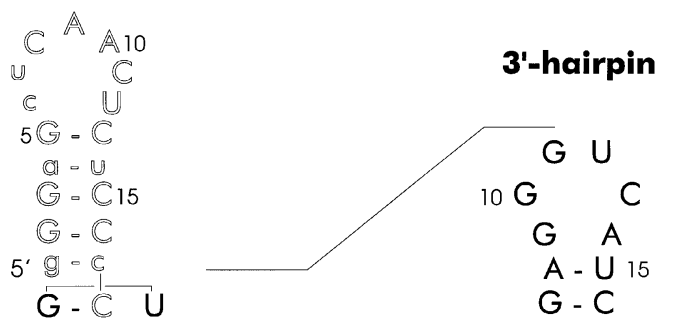

STEM 1 - $C$ C LOOP 2

$G-C$

C - $G$

$5 C-G$

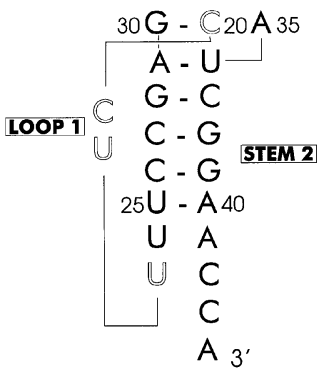

$U-A$

$\mathrm{U}-\mathrm{A} 20$

$g-C$

5. $9-C^{-}$

$\mathrm{A}_{3}$

Fig. 1. Secondary structures of the pseudoknotted domain found at the 3 '-terminus of TYMV genomic RNA (left) and of the 3 '-hairpin of the pseudoknot (right). Filled characters indicate the sequential coincidence of the two molecules. Non-wild-type residues are given in lower case.

pseudoknot formation would be reduced accordingly, leading to a destabilization of the intact pseudoknot. Hence, it is clear that the structure of the 3 '-hairpin loop strongly affects the assembly and stability of the entire pseudoknotted fragment.

In order to address this matter, we solved the threedimensional structure of the isolated 3'-hairpin of the pseudoknot (Figure 1) by NMR spectroscopy. The sequence of this hairpin loop matches that of the corresponding region in the pseudoknot. The results indicate that neither GA nor GC base pairs are formed in the hairpin loop. Nevertheless, the loop residues maintain a high structural integrity through an atypical conformation that is largely similar to the structure in the cognate pseudoknot. These findings emphasize the importance of loop conformations for the association into higher order structures in RNA molecules.

\section{Results}

Because of the excellent dispersion of nearly all resonances of the $3^{\prime}$-hairpin molecule, their assignment was possible without the aid of isotopically enriched RNA (Table I). Resonance identification proceeded in a straightforward manner, starting with a canonical anomeric to aromatic proton walk (Wijmenga et al., 1993). By this pattern of sequential NOEs, residues G1 through G11, and U12 through A23 could all be interconnected. All NOE connectivities agree with a regular helical conformation for the stem residues, and a propagation of the stacking pattern into the loop up to residue G10. Residues U12 and C13 also appear to stack in a normal fashion, but a number of irregular NOEs between A14 and U15, such as a connectivity between $\mathrm{A} 14(\mathrm{H} 8)$ and $\mathrm{U} 15\left(\mathrm{H}^{\prime}{ }^{\prime}\right)$, indicate an atypical conformation at this site. No NOEs are observed between G11 and U12, indicating an interruption of the stacking pattern at this position. An NOE-based sequential
Table I. Proton chemical shifts of the $3^{\prime}$-hairpin relative to TSP

\begin{tabular}{llllllll}
\hline & H8/H6 & H2/H5 & H1 $^{\prime}$ & H2 $^{\prime}$ & H3 $^{\prime}$ & H4' & im/am \\
\hline G1 & 8.20 & n.a. & 5.95 & 4.93 & 4.71 & 4.58 & 12.18 \\
G2 & 7.42 & n.a. & 5.83 & 4.54 & 4.18 & 4.55 & 13.24 \\
U3 & 7.79 & 5.13 & 5.60 & 4.53 & 4.48 & 4.11 & 14.41 \\
U4 & 8.00 & 5.62 & 5.67 & 4.53 & 4.49 & 4.45 & 13.89 \\
C5 & 7.84 & 5.67 & 5.51 & 4.31 & 4.45 & 4.10 & $8.41 / 7.00$ \\
C6 & 7.70 & 5.46 & 5.41 & 4.50 & 4.40 & 4.37 & $8.29 / 6.74$ \\
G7 & 7.51 & n.a. & 5.65 & 4.60 & 4.47 & 4.46 & 11.89 \\
A8 & 7.60 & 7.64 & 5.90 & 4.63 & 4.55 & 4.48 & \\
G9 & 7.09 & n.a. & 5.23 & 4.41 & 4.38 & 4.36 & $11.15 / 6.64$ \\
G10 & 7.38 & n.a. & 5.22 & 4.38 & 4.50 & 4.12 & $10.59^{\mathrm{a}}$ \\
G11 & 7.76 & n.a. & 5.65 & 4.83 & 4.66 & 4.38 & \\
U12 & 7.78 & 5.80 & 5.92 & 4.38 & 4.61 & & \\
C13 & 7.76 & 5.99 & 5.92 & 4.41 & 4.61 & & \\
A14 & 8.40 & 8.33 & 6.17 & 4.85 & 4.80 & 4.67 & \\
U15 & 7.96 & 5.81 & 5.35 & 4.51 & 4.05 & 4.44 & 14.15 \\
C16 & 7.86 & 5.70 & 5.61 & 4.56 & 4.44 & 4.46 & $8.25 / 6.85$ \\
G17 & 7.51 & n.a. & 5.67 & 4.61 & 4.53 & & 12.16 \\
G18 & 7.17 & n.a. & 5.67 & 4.56 & 4.51 & 4.47 & 12.39 \\
A19 & 7.68 & 7.10 & 5.88 & 4.56 & 4.66 & 4.50 & \\
A20 & 7.82 & 7.80 & 5.92 & 4.46 & 4.59 & 4.41 & \\
C21 & 7.47 & 5.16 & 5.39 & 4.22 & 4.34 & 4.36 & $8.40 / 7.00$ \\
C22 & 7.60 & 5.40 & 5.49 & 4.41 & 4.38 & 4.47 & $8.21 / 6.92$ \\
A23 & 8.03 & 7.36 & 5.98 & 4.05 & 4.30 & 4.25 & \\
\hline
\end{tabular}

${ }^{\mathrm{a}}$ Tentative assignment.

n.a., not applicable.

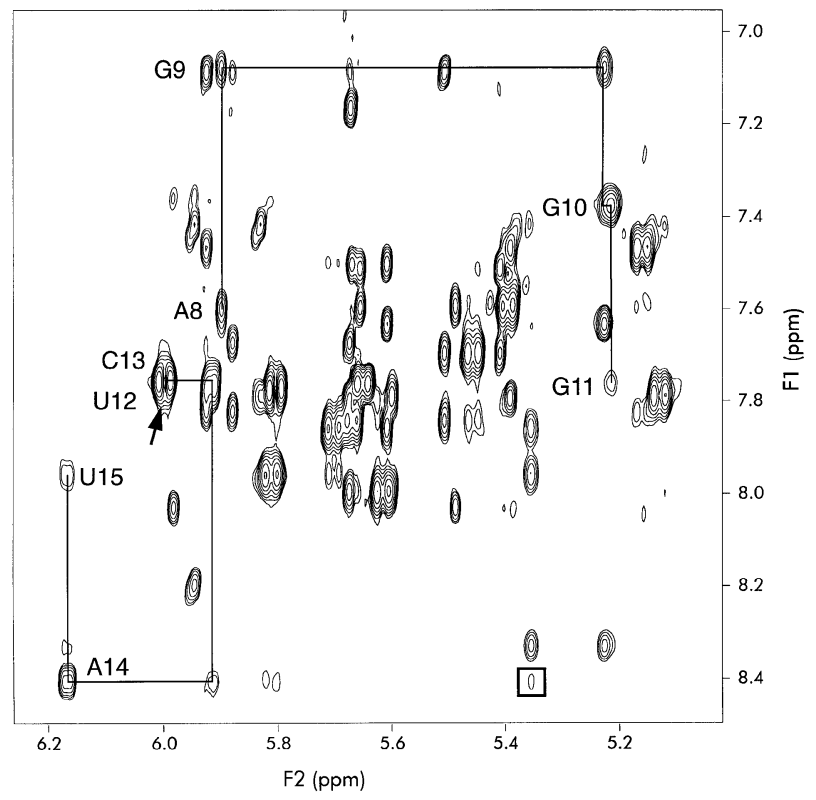

Fig. 2. Detail of a $240 \mathrm{~ms}$ NOESY spectrum recorded at $400 \mathrm{MHz}$, showing the anomeric to aromatic proton region. Sequential $\mathrm{H} 1^{\prime} \rightarrow \mathrm{H} 8 /$ H6 connectivities in the loop from A8 through G11 and from C13 through $\mathrm{U} 15$ are indicated by lines. Because of overlapping $\mathrm{H}^{\prime}$ resonances, $\mathrm{U} 12$ and $\mathrm{C} 13$ are connected by a sequential H6-H5 NOE (indicated by the arrow). The very unusual $3^{\prime} \rightarrow 5^{\prime}$ sequential $\mathrm{H} 1^{\prime}-\mathrm{H} 8$ NOE between U15 and A14 is boxed.

walk through the loop of the molecule is depicted in Figure 2.

A total number of $351 \mathrm{NOE}$-derived distance restraints could be collected for the 23-mer hairpin. The 141 interresidue distance restraints that were collected for the stem region all supported an A-type helical conformation. Fiftyone inter-residue restraints were obtained for the six loop residues and are shown in Figure 3. A number of the 


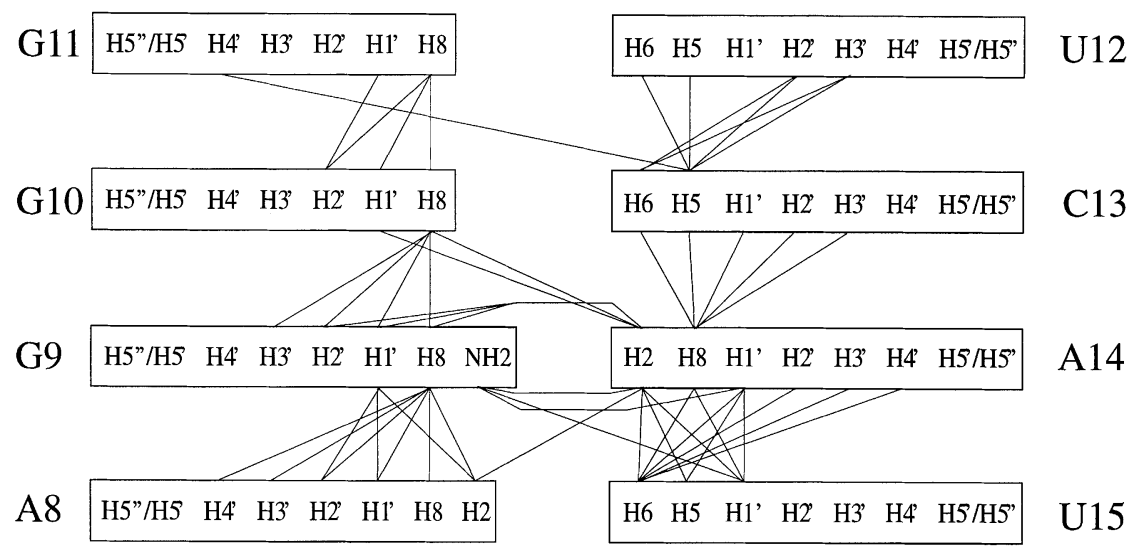

Fig. 3. Overview of the inter-residue NOEs (indicated by lines) found for the loop region.

latter are 'cross-strand', connecting the $\mathrm{H} 2$ proton of $\mathrm{A} 14$ to A8, G9 and G10, and indicative of A14 residing at the minor groove side of these latter residues. The kink in the backbone between G11 and U12 is substantiated by an unusual long-range NOE connecting the $\mathrm{H}^{\prime}$ of G11 and the $\mathrm{H} 5$ of $\mathrm{C} 13$.

Furthermore, several NOEs were observed from an exchangeable proton resonating at 6.64 p.p.m.: to a somewhat broadened imino proton resonance, assigned to G9, to the $\mathrm{H} 2$ proton of $\mathrm{A} 14$, and to the $\mathrm{H} 1{ }^{\prime}$ protons of $\mathrm{A} 14$ and U15. This resonance could be identified as the amino group of G9, since it was the only possible candidate of all amino and 2 '-hydroxyl protons in the loop to make the observed connectivities. Entering these restraints into the structure calculation improved the local r.m.s.d. but did not noticeably alter the average conformation.

\section{Loop structure}

The substantial amount of sequential and cross-strand distance restraints in both the stem and the loop region resulted in relatively high convergence rates in the structure calculations. More than half of the structures that were calculated starting from randomly extended conformations had the correct loop structure, and about one third had few violations and low r.m.s.ds to the average structure. Figure 4 depicts an overlay of the 10 best structures whose structural statistics are listed in Table II. Structures deviating markedly from the mean did so at the expense of many large violations, which were scattered throughout the molecule. These results exclude any ambiguity with respect to the solution to the experimental data set.

As was expected from the secondary structure and experimental data, residues $\mathrm{G} 1 \rightarrow \mathrm{A} 8$ and $\mathrm{U} 15 \rightarrow \mathrm{C} 22$ form regular Watson-Crick base pairs in an A-type helix. The 3 '-terminal residue A23 stacks on $\mathrm{C} 22$ and stabilizes the first base pair of the stem, as confirmed by the observed sharp imino proton resonance of G1. At the other side of the molecule, residue G9 stacks firmly on A8 (Figure 5), but it is slightly twisted towards the major groove, as a result of which it covers most of the underlying base moiety of A8. The stacking pattern is continued by G10, which is somewhat more flexible based on the local r.m.s.d. for this residue.

The more or less helical course of the nucleotide chain is interrupted around G11. Despite its considerable amount of motional freedom, this residue has a $\chi$-angle in the

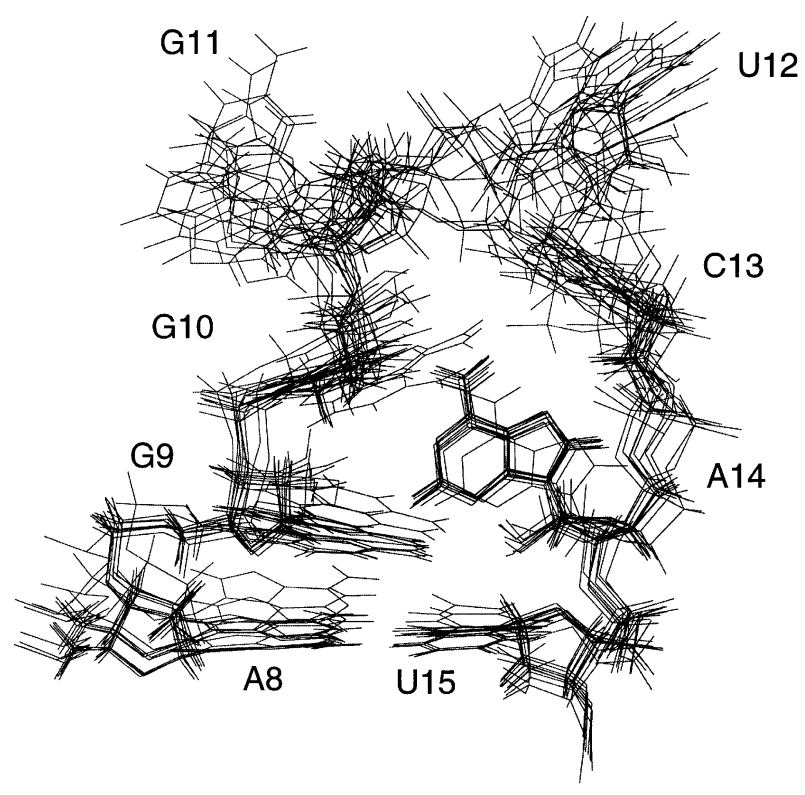

Fig. 4. Final ensemble of 10 lowest energy structures obtained by superimposing loop residues G9, G10, U12, C13 and A14. Only the loop region and the closing base pair are shown.

unusual syn-domain, facilitated by a sugar pucker which is largely of the S-type and a hydrogen bond from its $\mathrm{O} 4{ }^{\prime}$-atom to the $2^{\prime}$-hydroxyl of G10 [based on distances according to Saenger (1984) in the average structure of the calculated ensemble]. In addition, it is suggested by the ensemble of structures that the amino protons of G11 lie partially within hydrogen bonding distance to the $5^{\prime}$ phosphate oxygens, which may compensate for the reduced stacking energy of this residue. Interestingly, largely similar orientations have been observed earlier in a circular RNA trinucleotide (Mooren et al., 1994), and more recently in the six-membered loop of the iron responsive element (IRE; Addess et al., 1997). Thus, they may represent a preferred conformation for guanosines involved in redirecting the backbone in RNA loops.

Following the near inversion of the chain direction around $\mathrm{G} 11$, residues $\mathrm{U} 12$ and $\mathrm{C} 13$ stack partially and hover over A14, which is the central residue of the loop. This adenosine is tilted to an angle of $\sim 100^{\circ}$ with respect to the bases of G9 and G10. Since these are somewhat turned away, the adenosine base faces their minor groove 
Table II. Structural statistics of the final ensemble of 10 structures

\begin{tabular}{|c|c|c|c|}
\hline & All & Loop $^{a}$ & \\
\hline \multicolumn{4}{|l|}{ Distance restraints } \\
\hline intranucleotide & 159 & 65 & \\
\hline internucleotide & 192 & 51 & \\
\hline \multicolumn{4}{|l|}{ R.m.s.ds $\left(\AA{ }^{\circ}{ }^{\circ}\right)$} \\
\hline distance restraints (351) & $0.077 \pm 0.001$ & 0.063 & \pm 0.001 \\
\hline dihedral restraints (143) & $1.329 \pm 0.067$ & 1.149 & \pm 0.225 \\
\hline \multicolumn{4}{|l|}{ R.m.s.d. from idealized geometry } \\
\hline bonds $(\AA)$ & $0.0124 \pm 0.0006$ & 0.0115 & \pm 0.0003 \\
\hline angles $\left({ }^{\circ}\right)$ & $2.055 \pm 0.119$ & 1.837 & \pm 0.045 \\
\hline impropers $\left({ }^{\circ}\right)$ & $0.836 \pm 0.011$ & 0.231 & \pm 0.057 \\
\hline \multicolumn{4}{|l|}{ Restraint violations } \\
\hline number of distance violations ${ }^{\mathrm{b}}>0.3 \AA$ & \pm 1 & 2 & \pm 1 \\
\hline number of dihedral violations $\mathrm{c}^{\mathrm{c}}>3^{\circ}$ & \pm 1 & 2 & \pm 1 \\
\hline Atomic r.m.s.ds $(\AA)^{\mathrm{d}}$ & $1.65 \pm 0.52$ & 0.97 & \pm 0.19 \\
\hline
\end{tabular}

aResidues 9-14.

${ }^{\mathrm{b}}$ None $>0.4 \AA$.

${ }^{\mathrm{c}}$ None $>5^{\circ}$.

${ }^{\mathrm{d}}$ With respect to the average structure.

sides and participates in two hydrogen bonds: from the G9 amino group to the $\mathrm{N} 3$ of the adenosine, and from A14 amino to the $4^{\prime}$ of the sugar of G10 (though we cannot exclude the possibility of a hydrogen bond to the $\mathrm{O} 2$ ' of G10). The strain induced by this irregular orientation of A14 is also apparent from its sugar pucker, which is largely of the S-type.

The unusual orientation of A14 is supported by several observations. First, there is the large amount of sequential and long-range distance restraints from A14 to residues A8, G9, G10, C13 and U15, which to a large extent determine the high convergence rate that was obtained in the structure calculations, and the relatively low local r.m.s.d. Moreover, this set of 24 inter-residue NOEs is internally fully consistent and there is no evidence of significant local mobility for any of these nucleotides. Finally, the observed resonance for the G9 amino protons and their NOEs strongly suggest that these protons, which generally have severely broadened resonances, are involved in hydrogen bonding.

\section{Discussion}

As is visible in Figure 5, the stability in the loop is brought about through base-base stacking interactions (between A8, G9 and G10 and to a lesser extent between U12 and C13) and hydrogen bonding interactions in which four out of six loop residues are participating. The G11 amino-to-phosphate interaction supplements the hydrogen bond between the $2{ }^{\prime}-\mathrm{OH}$ group of $\mathrm{G} 10$ and the $\mathrm{O}^{4}$ '-atom of G11 in an energetical foundation for the turn in the RNA backbone. This is principally analogous to the $2^{\prime}-\mathrm{OH}$ hydrogen bonds which enforce a reversed $\pi$-turn in the central hairpin of the HDV ribozyme (Kolk et al., 1997), and it emphasizes the important role of the $2^{\prime}-\mathrm{OH}$ groups in RNA structure.

One might wonder why planar base pairs are not formed in this loop. As was mentioned in the Introduction, the formation of a G9-A14 base pair would seem probable on the basis of the loop sequence, or one could envision $\mathrm{C} 13$ base pairing to one of its opposing $\mathrm{G}$ residues. Part of the explanation for the absence of such interactions

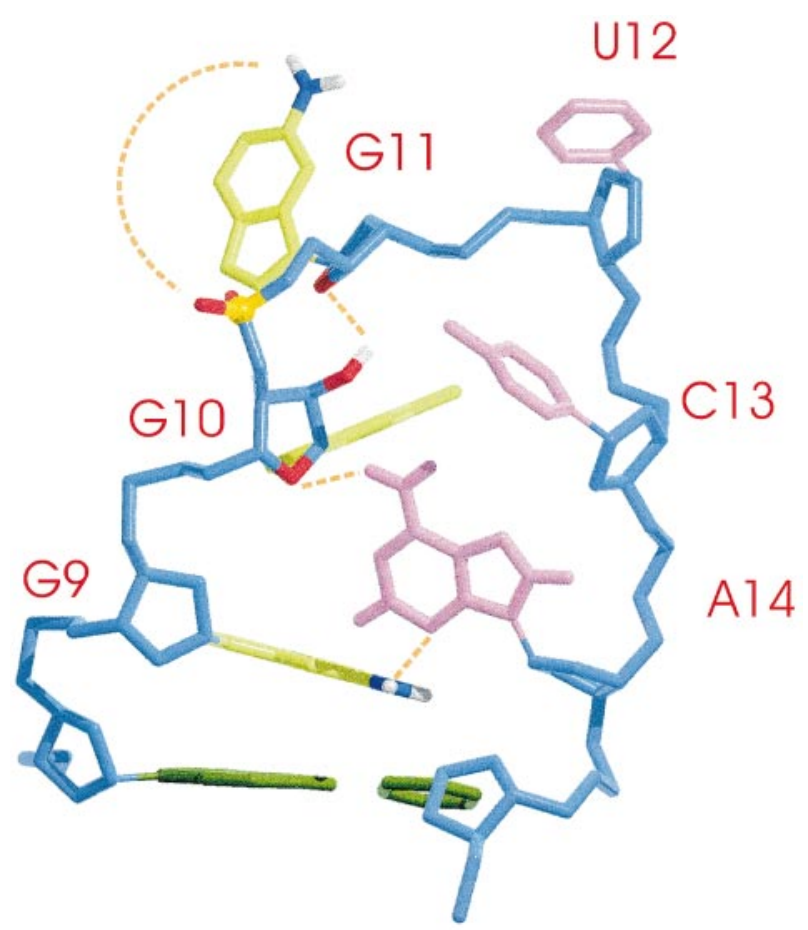

Fig. 5. Detail of the structure closest to the average of the final ensemble. Hydrogen bonds are indicated by dashed lines. The amino group of the flexible nucleotide G11 comes within hydrogen bonding distance to its $5^{\prime}$ phosphate oxygens in part of the ensemble (depicted as a curved dashed line). Color figures were generated using MOLMOL (Koradi et al., 1996).

follows from the evident stability of the present structure, which is governed by hydrogen bonds inconsistent with the suggested planar base pairs. Furthermore, the formation of a possible GA base pair is restrained on account of geometrical considerations, by which such base pairing is less favorable if a purine residue is preceding the guanosine (Serra et al., 1993; Hilbers et al., 1994). Alternatively, base pairing of $\mathrm{C} 13$ to either G10 or G11 would presumably lead to considerable strain around U12, while it is expected that the requirement for the purine base of A14 bulging 


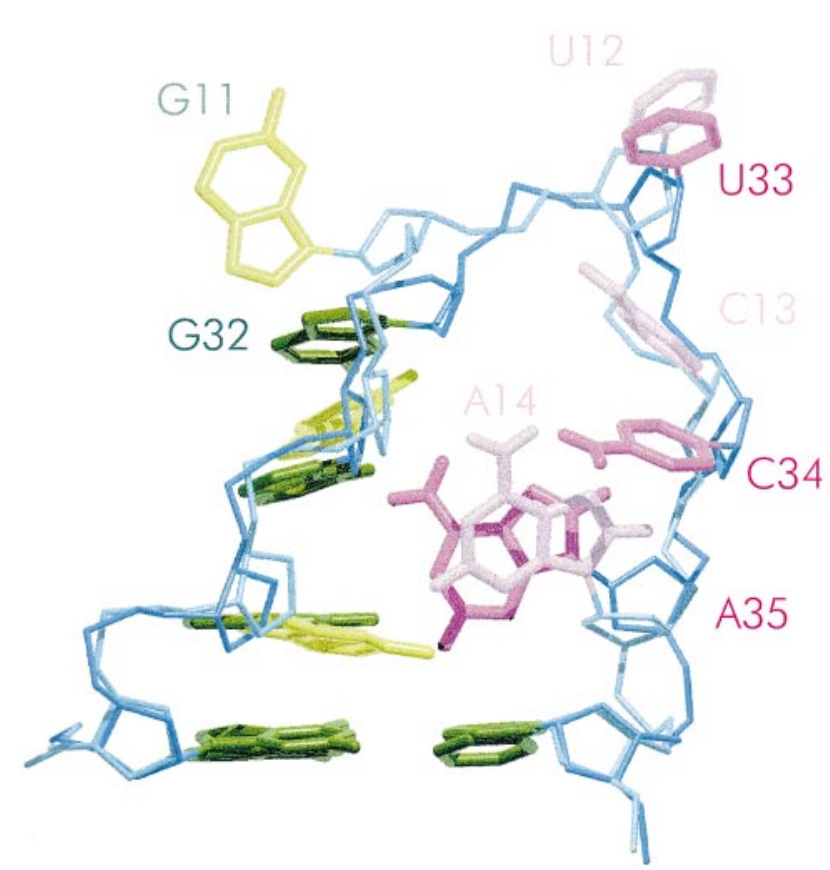

Fig. 6. Superposition of the $3^{\prime}$-hairpin loop (colored in a lighter shade) with the corresponding region of the pseudoknot (dark shade). Residue numbering in the two molecules is colored correspondingly.

out of the loop in the case of a G9-C13 base pair would unfavorably affect the stability of that configuration.

\section{Similarities to the pseudoknot structure}

The experimental data indicate a strong resemblance between the structure of the $3^{\prime}$-hairpin and that of the corresponding region in the pseudoknot. First, most of the cross-strand NOEs from the $\mathrm{H} 2$ proton of A14 are also found in the pseudoknot. In particular, those that connect the $\mathrm{H} 2$ to $\mathrm{H}^{\prime}$ and $\mathrm{H} 2$ ' of $\mathrm{G} 9$, which inherently deny the presence of a GA base pair at this position, are observed for both molecules. Secondly, a sugar pucker which is predominantly of the S-type was found for the adenosine in both cases. Finally, the aromatic proton chemical shifts, which are the most sensitive towards the relative orientation of the base moieties (Wijmenga et al., 1997), compare very well for the region around the central adenosine. Most convincingly, the $\mathrm{H} 8 / \mathrm{H} 6 / \mathrm{H} 2$ chemical shifts of residues G9, C13 and A14, which are all clearly shifted from the A-helical realm, deviate by $<0.2$ p.p.m. from the corresponding values in the pseudoknot.

These obvious similarities in experimental findings are also transparent in the structures. Figure 6 shows that most residues in the loop superimpose rather well with those in the pseudoknot, G11 being the only exception, since this guanosine is evidently not in a $\chi$-syn conformation when present in the stem 1 region of the pseudoknot. Interestingly, the deviating orientation of G11 in the hairpin relieves some of the strain that is present in loop 2 of the pseudoknot, where the sugar pucker of the adjacent uridine is predominantly of the S-type, as opposed to the equilibrium of $\mathrm{N}$ - and $\mathrm{S}$-type sugar puckers observed for $\mathrm{U} 12$ in the hairpin.

The relative flexibility of $\mathrm{C} 13$ in the $3^{\prime}$-hairpin as well as in the pseudoknot makes it difficult to interpret the importance of the dissimilarity in their orientations
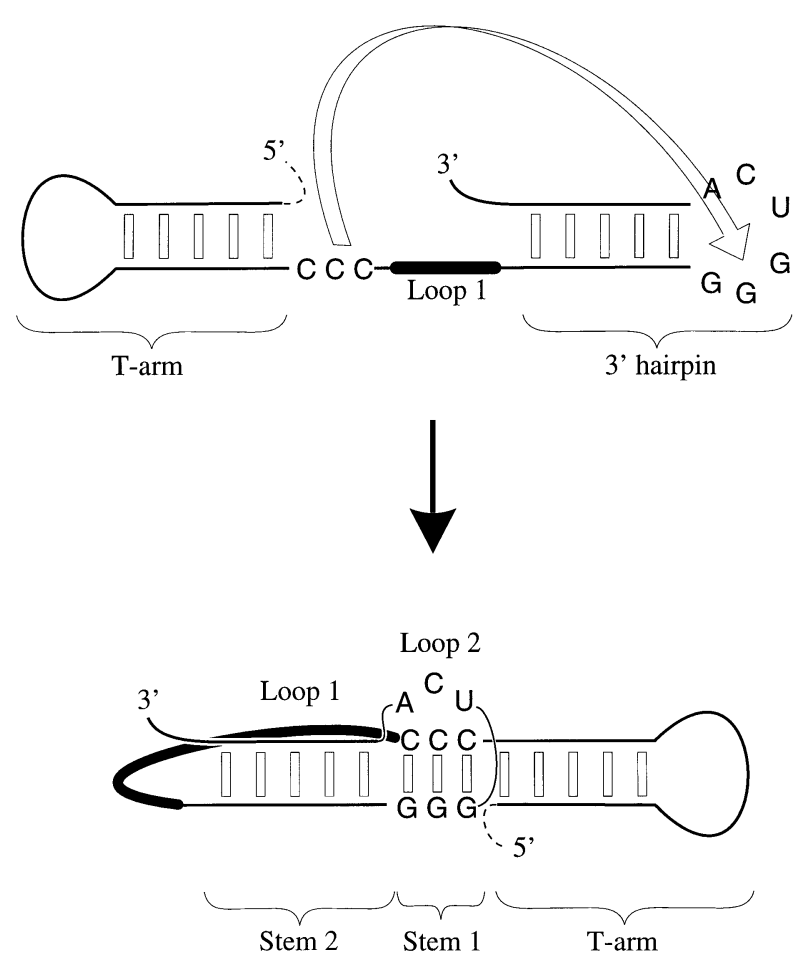

Fig. 7. Model of the folding mechanism for the pseudoknot and T-arm of TYMV RNA. The T-arm and 3'-hairpin are believed to be formed first (top; see text), after which the triple C-strand interacts with the preformed 3'-hairpin loop (bottom). In the complete tRNA-like structure, the anticodon arm is located at the $5^{\prime}$-side of the sequence (indicated by a dashed line).

between the two average structures. In the pseudoknot, this cytidine partly stacks on the adenosine, allowing its amino protons to come within hydrogen bonding distance to the $2^{\prime}-\mathrm{OH}$ of $\mathrm{G} 31$ (which is the counterpart of G10 in the hairpin molecule). In the hairpin, such an orientation may be obstructed by the base moiety of A14, which is in a slightly different orientation as compared with A35 in the pseudoknot.

The analogy between the two structures is most manifest for the G9-G10-A14 triad. At the central domain of the pseudoknot, the adenosine is harbored in the minor groove of stem 1 through an overtwisting around G30. Likewise, G9 is twisted towards the major groove of the hairpin loop, which sets A14 in an orientation very similar to that of A35 in the pseudoknot, despite the absence of a third strand. Although the central adenosine is not exactly in the same position in the two structures, which results in different hydrogen bonds to this residue, it is in both cases involved in tertiary interactions at the minor groove side of two guanosines, and a smooth interconversion between these two situations is expected to be feasible.

\section{Predetermination for pseudoknot formation}

The strong structural resemblance of the loop of the $3^{\prime}$ hairpin to the central region of the pseudoknot indicates that it is preformed and prone to combine with the $-\mathrm{C}_{18} \mathrm{C}_{19} \mathrm{C}_{20}$ - strand to produce stem 1 of the pseudoknot. This model differs markedly from the 'induced-fit' interactions which appear to govern RNA-protein binding (Aboul-ela et al., 1996; Allain et al., 1996; Battiste et al., 1996; Peterson and Feigon, 1996). The reason for this variation is unclear, and it remains to be established 
whether there is any systematic distinction between the two mechanisms.

The high structural integrity as well as the observed preformation in the loop make the $3^{\prime}$-hairpin a very plausible intermediate structure in the mechanism of pseudoknot folding. Thus, the formation of the T- and acceptor arm of the TYMV tRNA-like structure is expected to proceed as follows (Figure 7): (i) the RNA folds into a structure containing two adjacent hairpins (the T-arm and the $3^{\prime}$-hairpin, which contains stem 2 of the pseudoknot), separated by the triple $\mathrm{C}$ sequence and the future pseudoknot loop 1. The former are the two elements of secondary structure most likely to be formed first since they both contain a stable loop and a stem of five base pairs, and because hairpin structures are generally formed almost instantaneously in the kinetics of RNA folding. (ii) The 5'-half of the structure folds over the major groove of the stem of the $3^{\prime}$-hairpin to yield, after some minor rearrangement, the pseudoknot's second stem which, as has been mentioned above, is facilitated by the preformed structure of the 3 '-hairpin. Simultaneously, this part of the pseudoknot is stabilized by the stacking of the T-hairpin on the newly formed second pseudoknot stem. Hence, it appears that the role of the T-arm in the overall structure is twofold: it is a prerequisite for the stability of the pseudoknot domain (Kolk et al., 1998) and an additional driving force in the formation thereof.

\section{Materials and methods}

\section{Sample preparation}

The 23-mer RNA was prepared through in vitro transcription using T7 RNA polymerase (Milligan et al., 1987) with linearized plasmid as a template (Van Belkum et al., 1989). The product was purified using chromatography on Q-Sepharose and preparative polyacrylamide gel electrophoresis. The purified RNA was then dialyzed in the NMR buffer (20 mM sodium phosphate $\mathrm{pH} 6.8,0.1 \mathrm{mM}$ EDTA) and subsequently concentrated to $500 \mu \mathrm{l}$ using a Centricon microconcentrator to a final sample concentration of $\sim 3.5 \mathrm{mM}$. No $\mathrm{Mg}^{2+}$ was added to the sample, since this was shown to result in aggregation of the RNA.

\section{NMR methods}

All spectra were recorded on Bruker AM 400 and AMX 600 spectrometers, at $2^{\circ} \mathrm{C}$ for samples in $\mathrm{H}_{2} \mathrm{O}$, and at $22^{\circ} \mathrm{C}$ for those in $\mathrm{D}_{2} \mathrm{O}$. Exchangeable protons were assigned from 2D NOESY (Jeener et al., 1979) experiments which were executed using a jump return sequence (Plateau and Guéron, 1982) for water suppression. Other protons were assigned from 2D NOESY spectra in $\mathrm{D}_{2} \mathrm{O}$ employing mixing times of 80, 160 and $240 \mathrm{~ms}$, and from DQF-COSY (Shaka and Freeman, 1983) and TOCSY (Griesinger et al., 1988) experiments. Most C8/C6/C2 and $\mathrm{C}^{\prime}$ resonances could be assigned from a natural abundance ${ }^{13} \mathrm{C}-{ }^{1} \mathrm{H}$ HMQC experiment (Bax et al., 1983), confirming the matching proton assignments.

\section{Structural restraints}

Distances relating to imino protons were conservatively estimated from a jr-NOESY spectrum with a mixing time of $200 \mathrm{~ms}$. Other interproton distances were derived predominantly from 2D NOESY spectra at $80 \mathrm{~ms}$. Care was taken to diminish the effects of spin-diffusion, in particular for the loop region. NOE cross-peak intensities involving non-exchangeable protons were converted into distance restraints using the relaxation matrix approach of the program NO2DI (Van de Ven et al., 1991), and the resulting distances were given $\pm 20 \%$ error bounds. Weak NOEs from experiments at higher mixing times were assigned upper bounds of $6 \AA$. Scalar couplings between $\mathrm{H}^{\prime}{ }^{\prime}$ and $\mathrm{H} 2^{\prime}$ were determined from a DQF-COSY experiment, and were converted to $\mathrm{N}$ - or S-type sugar puckers (using dihedral restraints) in case of small $(<3 \mathrm{~Hz})$ or high $\left(>8 \mathrm{~Hz}\right.$ ) values, respectively. The $\mathrm{H} 1^{\prime} / \mathrm{H} 2^{\prime} / \mathrm{H} 3^{\prime}$ to $\mathrm{H} 6 / \mathrm{H} 8 \mathrm{NOE}$ intensities are indicative of a $\chi$-angle in the anti-range for all residues except for G11, which has an $\mathrm{H}^{\prime}$ to $\mathrm{H} 8 \mathrm{NOE}$ which is stronger than the average
H5-H6 NOE cross-peak intensity, and a rather weak NOE from H2' to $\mathrm{H} 8$, indicating a conformation in the syn-range. This latter conformation was not restrained, however, since G11 appeared to be a rather flexible residue.

Residues 1-10 and 15-23 clearly adopt an A-helical conformation, evidenced by chemical shifts, sugar puckers and NOE connectivities. These residues were therefore assigned glycosidic and backbone torsion angles in accordance with canonical A-type RNA with error bounds of $15^{\circ}$ for stem residues, and $30^{\circ}$ for G9 and G10. Hydrogen bonding and planarity restraints were imposed for all residues involved in WatsonCrick base pairing, as demonstrated by the imino proton spectra and all observed NOEs and chemical shifts. No standard restraints were imposed for any of the backbone torsion angles between the sugar of G10 and that of U15, to ensure an unbiased course of the polynucleotide chain. For this domain, the angle $\varepsilon$ was set to $225 \pm 60^{\circ}$, in order to exclude the stereochemically forbidden gauche ${ }^{+}$region, and the angles $\alpha$ and $\zeta$ were restricted to $0 \pm 120^{\circ}$, since all related ${ }^{31} \mathrm{P}$ chemical shifts are found to have A-helical values from a ${ }^{31} \mathrm{P}-{ }^{1} \mathrm{H}$ HETCOR experiment (Sklenar et al., 1986).

\section{Structure calculation}

A set of 100 structures was calculated using the torsion angle dynamics (TAD) protocol (Stein et al., 1997) in X-PLOR (Brünger et al., 1992). The TAD cooling step in this protocol was increased to $90 \mathrm{ps}$ so as to obtain a higher convergence rate. Thirty-three percent of the obtained pool of structures had no distance and dihedral restraint violations $>0.5 \AA$ and $5^{\circ}$, respectively. Ten structures of this ensemble best-fitting the experimental data were selected for presentation, and their statistics are listed in Table II.

\section{Acknowledgements}

The NMR experiments were performed at the Netherlands Foundation of Chemical Research (SON) HF-NMR facility (Nijmegen, The Netherlands). This research was supported by SON and the Dutch Life Science Foundation (SLW) with financial aid from the Netherlands Organization of Scientific Research (NWO). H.A.H. was supported by a grant from the Royal Netherlands Academy of Arts and Sciences (KNAW).

\section{References}

Aboul-ela,F., Karn,J. and Varani,G. (1996) Structure of HIV-1 TAR RNA in the absence of ligands reveals a novel conformation of the trinucleotide bulge. Nucleic Acids Res., 24, 3974-3981.

Abramovitz,D.L. and Pyle,A.M. (1997) Remarkable morphological variability of a common RNA folding motif: the GNRA tetraloopreceptor interaction. J. Mol. Biol., 266, 493-506.

Addess,K.J., Basilion,J.P., Klausner,R.D., Rouault,T.A. and Pardi,A. (1997) Structure and dynamics of the iron responsive element RNA: implications for binding of the RNA by iron regulatory binding proteins. J. Mol. Biol., 274, 72-83.

Allain,F.H.-T., Gubser,C.C., Howe,P.W., Nagai,K., Neuhaus,D. and Varani,G. (1996) Specificity of ribonucleoprotein interaction determined by RNA folding during complex formulation. Nature, $\mathbf{3 8 0}$, 646-650.

Battiste,J.L., Mao,H., Rao,N.S., Tan,R., Muhandiram,D.R., Kay,L.E., Frankel,A.D. and Williamson,J.R. (1996) Alpha helix-RNA major groove recognition in an HIV-1 rev peptide-RRE RNA complex. Science, 273, 1547-1551.

Bax,A., Griffith,R. and Hawkins,B.L. (1983) Correlation of proton and nitrogen-15 chemical shifts by multiple quantum NMR. J. Magn. Reson., 55, 301-315.

Brünger,A.T. (1992) X-PLOR. A System for X-ray Crystallography and $N M R$. Yale University Press, New Haven, CT.

Cai,Z., Gorin,A., Frederick,R., Ye,X., Hu,W., Majumdar,A., Kettani,A. and Patel,D. (1998) Solution structure of P22 transcriptional antitermination N peptide-boxB RNA complex. Nature Struct. Biol., 5, 203-212.

Cate,J.H., Gooding,A.R., Podell,E., Zhou,K., Golden,B.L., Kundrot,C.E., Cech,T.R. and Doudna,J.A. (1996) Crystal structure of a group I ribozyme domain: principles of RNA packing. Science, 273, 16781685.

Griesinger,C., Otting,G., Wüthrich,K. and Ernst,R.R. (1988) Clean TOCSY for ${ }^{1} \mathrm{H}$ spin system identification in macromolecules. $J$. Am. Chem. Soc., 110, 7870-7872. 
Heus,H.A. and Pardi,A. (1991) Structural features that give rise to the unusual stability of RNA hairpins containing GNRA loops. Science, 253, 191-194.

Hilbers,C.W., Heus,H.A., Van Dongen,M.J.P. and Wijmenga,S.S. (1994) The hairpin elements of nucleic acid structure: DNA and RNA folding. In Eckstein,F. and Lilley,D. (eds), Nucleic Acids and Molecular Biology. Vol. 8. Springer-Verlag, Berlin, Germany, pp. 56-104.

Jeener,J., Meier,B.H., Bachmann,P. and Ernst,R.R. (1979) Investigation of exchange processes by two-dimensional NMR spectroscopy. J. Chem. Phys., 71, 4546-4553.

Jucker,F.M. and Pardi,A. (1995) GNRA tetraloops make a U-turn. RNA, 1, 219-222.

Kolk,M.H., Heus,H.A. and Hilbers,C.W. (1997) The structure of the isolated, central hairpin of the HDV antigenomic ribozyme. Novel structural features and similarity of the loop in the ribozyme and free in solution. EMBO J., 16, 3685-3692.

Kolk,M.H., Van der Graaf,M., Wijmenga,S.S., Pleij,C.W.A., Heus,H.A. and Hilbers,C.W. (1998) NMR structure of a classical RNA pseudoknot: interplay of single- and double-stranded RNA. Science, 280, 434-438.

Koradi,R., Billeter,M. and Wüthrich,K., (1996) MOLMOL: a program for display and analysis of macromolecular structures. J. Mol. Graph., 14, 51-55.

Legault,P., Li,J., Mogridge,J., Kay,L.E. and Greenblatt,J. (1998) NMR structure of the bacteriophage $\lambda \mathrm{N}$ peptide/boxB RNA complex: recognition of a GNRA fold by an arginine-rich motif. Cell, 93, 289-299.

Milligan,J.F., Groebe,D.R., Witherell,G.W. and Uhlenbeck,O.C. (1987) Oligoribonucleotide synthesis using T7 RNA polymerase and synthetic DNA templates. Nucleic Acids Res., 15, 8783-8798.

Mooren,M.M., Wijmenga,S.S., van der Marel,G.A., van Boom,J.H. and Hilbers,C.W. (1994) The solution structure of the circular trinucleotide cr(GpGpGp) determined by NMR and molecular mechanics calculation. Nucleic Acids Res., 22, 2658-2666.

Oubridge,C., Ito,N., Evans,P.R., Teo,C.H. and Nagai,K. (1994) Crystal structure at $1.92 \AA$ resolution of the RNA-binding domain of the U1A spliceosomal protein complexed with an RNA hairpin. Nature, 372, 432-438.

Peterson,R.D. and Feigon,J. (1996) Structural change in Rev responsive element RNA of HIV-1 on binding Rev peptide. J. Mol. Biol., 264, 863-877.

Plateau,P. and Guéron,M. (1982) Exchangeable proton NMR without base-line distortion, using new strong-pulse sequences. J. Am. Chem. Soc., 104, 7310-7311.

Puglisi,J.D., Tan,R., Calnan,B.J., Frankel,A.D. and Williamson,J.R. (1992) Conformation of the TAR RNA-arginine complex by NMR spectroscopy. Science, 257, 76-80.

Saenger,W. (1984) Principles of Nucleic Acid Structure. Springer-Verlag, New York, NY.

Serra,M.J., Lyttle,M.H., Axenson,T.J., Schadt,C.A. and Turner,D.H. (1993) RNA hairpin loop stability depends on closing base pair. Nucleic Acids Res., 21, 3845-3849.

Shaka,A.J. and Freeman,R. (1983) Simplification of NMR spectra by filtration through multiple-quantum coherence. J. Magn. Reson., 51, 169-173.

Sklenar,V., Miyashiro,H., Zon,G., Miles,H.T. and Bax,A. (1986) Assignment of the $31 \mathrm{P}$ and $1 \mathrm{H}$ resonances in oligonucleotides by twodimensional NMR spectroscopy. FEBS Lett., 208, 94-98.

Stein,E.G., Rice,L.M. and Brünger,A.T. (1997) Torsion-angle dynamics as a new efficient tool for NMR structure calculation. J. Magn. Reson., 124, 154-164.

Valegård,K., Murray,J.B., Stockley,P.G., Stonehouse,H.J. and Liljas,L. (1994) Crystal structure of an RNA bacteriophage coat proteinoperator complex. Nature, 371, 623-626.

Van Belkum,A., Wiersema,P.J., Joordens,J., Pleij,C.W.A., Hilbers,C.W. and Bosch,L. (1989) Biochemical and biophysical analysis of pseudoknot-containing RNA fragments. Melting studies and NMR spectroscopy. Eur. J. Biochem., 183, 591-601.

Van de Ven,F.J.M., Blommers,M.J.J., Schouten,R.E. and Hilbers,C.W. (1991) Calculation of interproton distances from NOE intensities. A relaxation matrix approach without requirement of a molecular model. J. Magn. Reson., 94, 140-151.

Wijmenga,S.S., Mooren,M.W. and Hilbers,C.W. (1993) NMR of nucleic acids; from spectrum to structure. In Roberts,G.C.K. (ed.), NMR of Macromolecules. A Practical Approach. Oxford University Press, New York, NY, pp. 217-288.
Wijmenga,S.S., Kruithof,M. and Hilbers,C.W. (1997) Analysis of ${ }^{1} \mathrm{H}$ chemical shifts in DNA. Assessment of the reliability of ${ }^{1} \mathrm{H}$ chemical shift calculations for use in structure refinement. J. Biomol. NMR, 10, $337-350$.

Received July 20, 1998; revised October 14, 1998; accepted October 15, 1998 